\title{
Morphological parameters of fourth lumbar spinous process palpation: a three- dimensional reconstruction of computed tomography
}

Qi Feng ${ }^{1,2+}$, Lei Zhang ${ }^{3,4,2^{*+}}$, Mengyao Zhang ${ }^{1,2+}$, Youliang Wen ${ }^{5}$, Ping Zhang ${ }^{6}$, Yi Wang ${ }^{7,2}$, Yan Zeng ${ }^{8}$ and Junqiu Wang ${ }^{7,2}$

\begin{abstract}
Background: The localization of lumbar fourth spinous process (L4-SP) is an important anatomical landmark, and identifying its accurate position is essential for the diagnosis and treatment of waist diseases.

Methods: Five hundred participants were scanned with positive and lateral computed tomography (CT), which aimed to clarify anatomic characteristics of L4-SP. Anatomical parameters of the surface localization of L4-SP were measured and recorded through a three-dimensional (3D) reconstruction.

Results: Five hundred participants were classified into three types according to the position of BC with the iliac spine. There are just 266 that the line between the highest point of the iliac spine on both sides located on L4-SP (type I, 53.20\%), 16 above L4-SP (type II, 3.20\%), and 218 below L4-SP (type III, 43.60\%). BC in type I (15.92 \pm 1.30 $\mathrm{mm})$ is longer than type III $(15.56 \pm 1.32 \mathrm{~mm})$. While the angle combined with $A B$ and $B C$ is different in the three groups, the angle in type I $\left(173.00 \pm 4.83^{\circ}\right)$ is larger than that in type II $\left(164.69 \pm 5.50^{\circ}\right)$ and type III $\left(159.45 \pm 8.39^{\circ}\right)$. Other measurements were not found any significant differences between above.
\end{abstract}

Conclusion: The traditional palpation for L4-SP is not absolutely exact. The accuracy rate is only $53.20 \%$, and the errors may cause serious consequences.

Keywords: Palpation, Spine, Lumbar vertebrae, Imaging, Three-dimensional

\section{Background}

Lumbar fourth spinous process (L4-SP) is in the connection between the highest point on both sides of the posterior superior iliac spine (PSIS) with its surface localization relates to the examination, diagnosis, and treatment of many lumbar diseases [1]. Clinical

\footnotetext{
*Correspondence: zhanglei870722@126.com

${ }^{\dagger}$ Qi Feng, Lei Zhang and Mengyao Zhang contributed equally to this work.

${ }^{3}$ Department of Orthopedics, Affiliated Traditional Chinese Medicine Hospital of Southwest Medical University, Luzhou 646000, China

${ }^{4}$ National Key Discipline of Human Anatomy, School of Basic Medical Sciences, Southern Medical University, Guangzhou, China

Full list of author information is available at the end of the article
}

operations often find body position line-the highest point on both sides of PSIS to find the exact location of L4 [2]. Some scholars deal with L4-SP are performed frequently, including lumbar puncture and spinal anesthesia (SA). The results of degenerative changes of lumbar intervertebral disk increased with age until the incidence rate over $90 \%$ after the age of 50 , and at the same time, lumbar disk herniation has a lifetime incidence of $1-2 \%$, especially in the L4-L5 and L5-S1 [1, 35]. After that, both conservative treatment and surgical treatment of lumbar disk herniation are based on the exact location of L4. 
Lumbar puncture is wildly used in the central nervous system; the entry point of lumbar puncture is the line determined by the superior part of the iliac crest, and inserting the needle at the L3/4 or L4/5 intervertebral space is usually as an accepted practice. In addition, due to the inter-individual deviation of L4-SP, the identification of suitable approaches to the entry point is challenging, especially newborns, the elderly, and parturients $[6,7]$.

Furthermore, spinal anesthesia is a form of regional anesthesia involving injection of a local anesthetic into the subarachnoid space, which is a commonly used anesthetic technique, both alone and in combination with either sedation or general anesthesia. It also depends on the accurate surface location of L4-SP. Local anesthetics were injected into the subarachnoid space through the L3/L4 intervertebral space to block the conduction function of some spinal nerves and induce anesthesia in the corresponding dominant areas. Anatomically, these operations go from shallow to deep through the skin, spinous ligaments, interspinous ligament, ligamentum flavum, epidural space, and dura mater spinalis, with nerves surrounded. Above that, the wrong positioning will hurt these structures can lead serious consequences including paraplegia and headache. In that case, it was clear that adequate knowledge of L4 morphology is necessary for the spinal surgeon in order to avoid damage to the vertebral arteries, spinal cord, or nerve roots during fixation interventions involving the posterior cervical spine. However, the number of failed lumbar puncture cases remains high $[6,8,9]$. Among the rest, postdural puncture headache is the most common complication, which is caused by the leakage of CSF [10]. Meanwhile, it is reported that the incidence is up to $75 \%$.

L4-SP has high clinical value that can be demonstrated by all of those examples. However, the position of L4-SP is usually determined by the position of the highest point on both sides of PSIS. But the accuracy rate of palpation of L4-SP is 36\%, which is too low to locate the body surface position of L4-SP accurately enough [11-13]. Besides, only a few reports have assessed the interindividual variation of L4-SP. In order to understand the anatomy classification of L4-SP fully to improve the clinical effect, this research did numerical measure and statistic analysis of L4 on 500 subjects from the Chinese to investigate the relationship between L4-SP and body position line in tummy position by using $3 \mathrm{D}$ reconstruction. In that case, it could be helpful for improving the accuracy rate of L4-SP detection could be improved.

\section{Methods}

\section{Patients}

It should be excluded that 47 individuals with lumbar pelvic fracture, dysplasia, lumbar scoliosis, or other spinal pathologic abnormalities. Five hundred subjects, aging 18 to 75, participated in this study from 2014 to 2018. Due to the limited availability of data, sex, height, and weight were not included.

\section{Methods}

In CT scans, the location of 500 subjects is standardized to guarantee the relationship between body position line and L4-SP. In the anteroposterior and lateral lumbar images, carry on 3D reconstruction to remove L4-SP and adjust the appropriate proportion for choosing the correct position, to ensure accurate measurement results. The spiral computed tomography (CT) scanner (Somatom Emotion; Siemens AG, Munich, Germany) was used with the following scan conditions: voltage, $130 \mathrm{kV}$; current, $180 \mathrm{~mA}$; thickness, $0.75 \mathrm{~mm}$; and matrix size, $512 \times 512$. The $3 \mathrm{D}$ images were stored on the picture archiving communication system (PACS version 4.0; DJ Health Union Systems Corporation, Shanghai, China).

The parameters were measured 3 times and recorded carefully by a doctor with 5 years of experience in $3 \mathrm{D}$ printing work for avoiding intra-observer and interobserver variation. $\mathrm{AB}$, the distance between point $\mathrm{A}$ and point $B$ (point $A$ the $L 4$ spinous process, point $B$ the highest points of the right side of PSIS); $A C$, the distance between point $\mathrm{A}$ and point $\mathrm{C}$ (point $\mathrm{C}$ the highest points of the left side of PSIS); and $\mathrm{BC}$, the distance between point $B$ and point $C$ (Fig. 1). Angle-combined with $A B$ and $\mathrm{BC}$; what's more, in order to find out the impact of the own factors of L4-SP on the results, measured the angle between the axis of L4-SP and the median line of the vertebra in the superior aspect $(\angle \alpha)$ or in the lateral aspect $(\angle \gamma)$; and between the SP axis and the line attached the transverse processes $(\angle \beta)$ (Fig. 2). Lengththe length of SP. The accuracy rate of palpation of L4SP is $36 \%$ [11], and we adopted the same calculation: the number of spinous processes on $A B$ divided by sample size multiply by $100 \%$.

\section{Statistical analyses}

A statistical analysis was proceeding with SPSS, version 20.0 (IBM Corp., Armonk, NY, USA). According to the formula $N=Z \times P \times(1-\mathrm{P}) / \mathrm{E}$, the sample size can be figured out (where $N$ is a statistic, $Z=1.96$; $P$ is 0.5 as a probability value, $E$ is $5 \%$ as an error value, confidence level is 95\%). Through the above calculation, $N=500>$ 384 , and 500 scapulae could be adopted in the study. When it accorded with the normal distribution, the chi $^{2}$ test and one-way ANOVA were adopted, and all data were presented with the mean and standard error. In addition, there is a $P$ value $<0.05$ as statistically significant. 


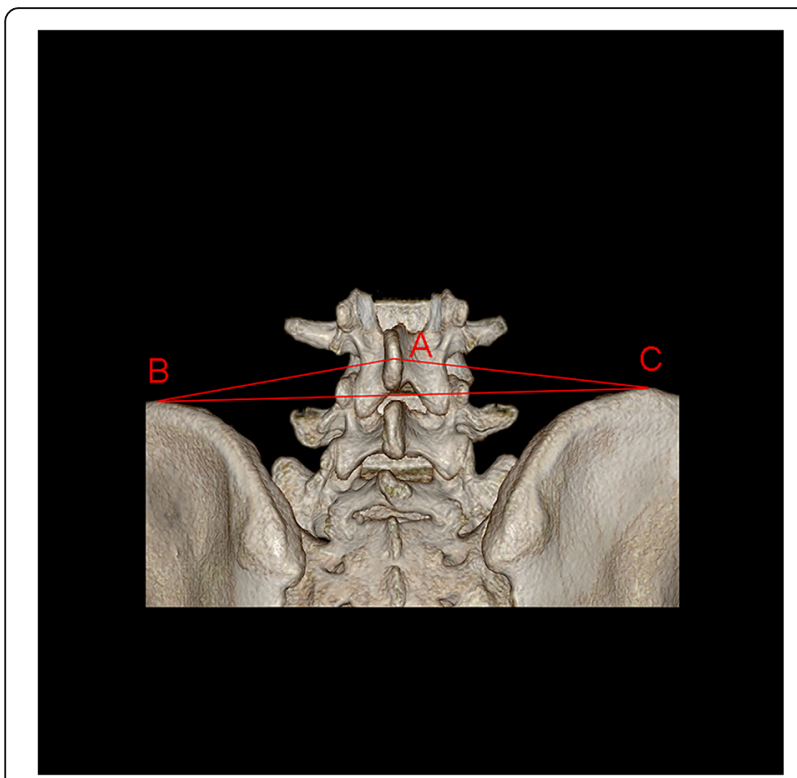

Fig. 1 Measurements between L4-SP and posterior superior iliac spine (PSIS). Point A, the L4 spinous process; point B, the highest points of right side of PSIS; point $C$, the highest points of left side of PSIS

\section{Ethics}

All procedures were approved by the Ethical Committee of Affiliated Traditional Chinese Medicine Hospital of Southwest Medical University (SWMCTCM2017-0801).

\section{Results}

Among 500 participants, three types were defined according to the distance of $\mathrm{BC}$ on the spine situating on different positions: 266 on L4-SP (type I, 53.20\%), 16 above L4-SP (type II, 3.20\%), and 218 below L4-SP (type III, 43.60\%). Type I is the most common one among the Chinese population. All data were normally distributed, and measured relative data of the L4 SP based on classification were recorded in Table 1.
Significant differences were also observed in $\mathrm{BC}$ and the angle combined with $\mathrm{AB}$ and $\mathrm{BC}$ among different types. $\mathrm{BC}$ in type $\mathrm{I}(15.92 \pm 1.30 \mathrm{~mm})$ is longer than type III $(15.56 \pm 1.32 \mathrm{~mm})$, while the angle combined with $\mathrm{AB}$ and $\mathrm{BC}$ in type $\mathrm{I}\left(173.00 \pm 4.83^{\circ}\right)$ is larger than that in type II $\left(164.69 \pm 5.50^{\circ}\right)$ and type III $\left(159.45 \pm 8.39^{\circ}\right)$. Comparing the length of the L4-SP to the left (AC) with the right $(\mathrm{AB})$, there were no different significant among three types, and the same consequences also happened to three angles: $\alpha, \beta, \gamma$. The number of spinous processes on $\mathrm{AB}$ was 266 , according to the calculation, the accurate palpation rate for L4-SP is $53.20 \%$.

\section{Discussion}

Lumbar disk herniation, lumbar puncture, and spinal anesthesia were usually common situations, which involved the surface localization of L4-SP. The accuracy in identifying L4 using manual palpation by clinicians is limited due to the inter-individual variation in the morphology of L4-SP [2]. Most studies focus on the mechanisms of diseases affecting it. Even though, there were only a few reports about the inter-individual variation of L4-SP. The objective of the present study was to explore the anatomical features of L4-SP, which may facilitate to find the exact insertion point.

All data about 500 participants were collected by 3D of CT construction from 2014 to 2018. All patients were Han Chinese and aged 18 to 75 . Thus, the information in this study had practical significance for the operation in China. By this way, Table 1 indicated that there were statistical differences in $\mathrm{BC}$ and the angles between three types. Among them 266 on L4-SP (type I, 53.20\%), 16 above L4-SP (type II, 3.20\%), and 218 below L4-SP (type III, $43.60 \%)$. This can be used to be one of the basis for determining the position of L4-SP. Unlike conventional $\mathrm{X}$-ray analysis, CT provides detailed images of numerous types of tissue as well as the bones and blood vessels, which is a rapid and accurate procedure. In that case, based on anatomical parameters form CT to obtain a $3 \mathrm{D}$

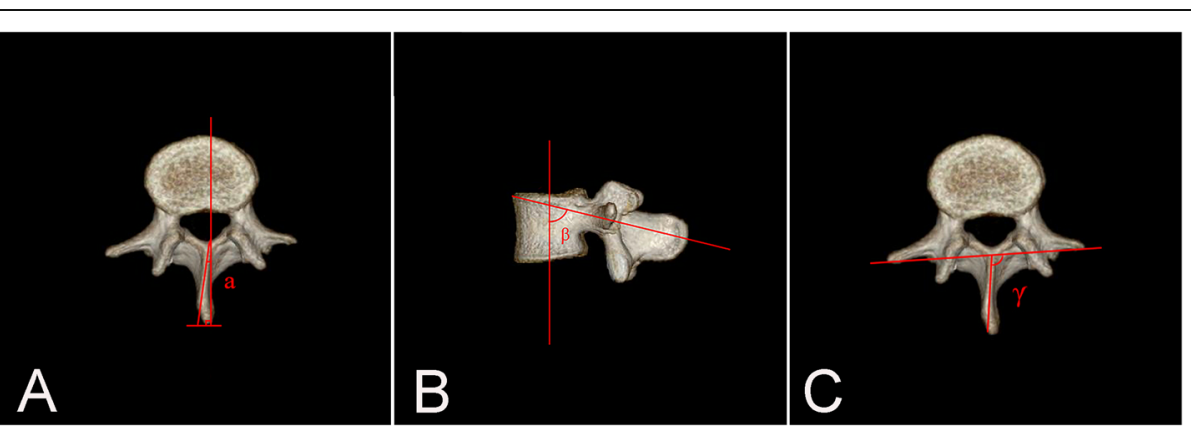

Fig. 2 Measurements of $L 4$. $\mathbf{a} \angle a$, the angle between the axis of L4-SP and the median line of the vertebra in the superior aspect. $\mathbf{b} \angle \beta$, the angle between the axis of L4-SP and the median line of the vertebra in the lateral aspect. $\mathbf{c} \angle \gamma$, the angle between the SP axis and the line attached the transverse processes 
Table 1 The parameters of the L4-SP

\begin{tabular}{llll}
\hline Distribution & Type I & Type II & Type III \\
\hline Numbers & 266 & 16 & 218 \\
Ratios (\%) & 53.20 & 3.20 & 43.60 \\
BC (mm) & $15.92 \pm 1.30^{\mathrm{b}}$ & $16.16 \pm 1.18$ & $15.56 \pm 1.32$ \\
AB (mm) & $8.02 \pm 0.73$ & $7.98 \pm 0.76$ & $7.89 \pm 0.79$ \\
AC (mm) & $7.96 \pm 0.79$ & $8.26 \pm 0.81$ & $7.97 \pm 0.84$ \\
Angle $\left(^{\circ}\right)$ & $173.00 \pm 4.83^{\mathrm{ab}}$ & $164.69 \pm 5.50^{\mathrm{b}}$ & $159.45 \pm 8.39$ \\
$\mathrm{a}\left({ }^{\circ}\right)$ & $1.07 \pm 3.05$ & $1.00 \pm 3.97$ & $1.18 \pm 3.33$ \\
$\beta\left(^{\circ}\right)$ & $89.78 \pm 5.28$ & $90.00 \pm 6.15$ & $89.58 \pm 3.44$ \\
$\left(^{\circ}\right)$ & $74.46 \pm 3.26$ & $74.38 \pm 3.65$ & $74.16 \pm 5.92$ \\
Length $(\mathrm{mm})$ & $3.06 \pm 0.41$ & $2.84 \pm 0.52$ & $3.07 \pm 0.51$
\end{tabular}

${ }^{a} P<0.05$ VS above the L4-SP

${ }^{\mathrm{b}} P<0.05$ VS below the L4-SP

model, they could locate it accurately and reduce the risk of operation $[3,5,9]$. When it came to some possible reasons, such as anatomic factors, the size of the lumbar spine changes with the development and the growth of human skeletons under the influence of genetic factors, and this kind of physiological diversity may affect the accuracy of palpation [4, 13-15]. Besides, degenerative changes occurring in the lumbar spine can be worse by the influence of patients' lifestyles. The most common and serious transform was lumbar disk herniation, in broken disk herniation and retrograde lumbar disk herniation, which would cause broken fibrous ring, cartilage endplate, and nucleus burst out, and then resulting in the height of intervertebral disk changes. In addition, lumbar spondylolisthesis also had high incidence and influence the palpation accuracy, especially in different positions or sports. It is important to find the highest points PSIS to determine the accurate position of L4-SP [16]. On the account of normal physiological factors, the traditional palpation operation is used for normal state. However, there are 8 participants in the study that have 6 lumbar vertebrae, which cannot be adopted because of abnormalities.

As to the clinic factor, the degree of cooperation between patients and doctors can be an influential factor. Different levels of mistakes when different positions of the examinees took on, even experienced physician may make. If the $3 \mathrm{D}$ of $\mathrm{CT}$ construction was used to verify the position of the vertebral levels in patients, the risk of the devastating and permanent complications resulting from spinal cord injection can be reduced. In that case, it was clear that adequate knowledge of L4 morphology is necessary for the spinal surgeon in order to avoid damage to the vertebral arteries, spinal cord, or nerve roots during fixation interventions involving the posterior cervical spine. Hence, it can be more safer for patients with abnormal spinal anatomy and the morbidly obese, particularly in those who have difficulties in palpating anatomical landmarks $[17,18]$. Therefore, there were a series of errors which may happen in clinical practice. The traditional palpation of L4-SP is not completely exact. The accuracy rate is only $53.20 \%$, and the errors may cause serious consequences [19].

Nevertheless, there are still some limitations. Though the accuracy of the fourth lumbar spinous process palpation can be improved by 3D of CT reconstruction, the operative level of clinician is decisive. Above that, the samples in this research are limited to the southwest, and the sample size is not so adequate now. The image resources are limited when it comes to costs and radiation because it is far more practical that patients' willingness to use radiographs, computed tomography, and MRI are reduced $[18,20]$.

\section{Conclusion}

Among 500 participants, three types were defined according to the distance of $\mathrm{BC}$ on the spine situating on different positions. Among three types, type I is the most common one among the Chinese population. As an important position of the body, the accuracy rate of palpation of L4-SP has high clinical value. However, it is too low to locate the body surface position of L4 accurately enough now. This study demonstrated that the anatomy of L4 can be understood fully through 3D reconstruction technology and improve the clinical effect, so that the accuracy of SP' detection can be authoritative.

\section{Abbreviations \\ L4-SP: Lumbar fourth spinous process; CT: Computed tomography; 3D: Three-dimensional; PSIS: Posterior superior iliac spine; SA: Spinal anesthesia}

\section{Acknowledgements}

The authors wanted to show their gratitude to the imaging department in Southwest Medical University that provided the samples.

\section{Authors' contributions}

Qi Feng: conception and design, edit, and process articles; Lei Zhang: conception, design and instruction; Mengyao Zhang: edit and process articles; Youliang Wen: picture data processing and statistical analysis; Ping Zhang: edit and process articles; Yi Wang: picture data processing and statistical analysis; Yan Zeng: data collection and literature search; Junqiu Wang: data collection and literature search. All authors read and approved the final manuscript.

\section{Funding}

Luzhou Municipal Government-Southwest Medical University Science and Technology Strategic Cooperation Project in 2018 (2018LZXNYD-ZK43); Luzhou Science and Technology Innovation Seedling Cultivation Project in 2018 (2018-RCM-72); and Academician Workstation Construction Project of Luzhou (20180101).

\section{Availability of data and materials}

The datasets used and analyzed during the current study are available from the corresponding author on reasonable request.

Ethics approval and consent to participate

All procedures were approved by the Ethical Committee of Affiliated Traditional Chinese Medicine Hospital of Southwest Medical University 
(SWMCTCM2017-0801). All patients signed a general consent of the Ethical Committee of Affiliated Traditional Chinese Medicine Hospital of Southwest Medical University for using and publishing their data for scientific use.

\section{Competing interests}

No conflict of interest exists in the submission of this manuscript, and the manuscript is approved by all authors for publication.

\section{Author details}

${ }^{1}$ School of Clinical Medicine, Southwest Medical University, Luzhou, China. ${ }^{2}$ Academician Workstation in Luzhou, Luzhou, China. ${ }^{3}$ Department of Orthopedics, Affiliated Traditional Chinese Medicine Hospital of Southwest Medical University, Luzhou 646000, China. ${ }^{4}$ National Key Discipline of Human Anatomy, School of Basic Medical Sciences, Southern Medical University, Guangzhou, China. ${ }^{5}$ School of Rehabilitation Medicine, Gannan Medical University, Ganzhou, China. ${ }^{6}$ Operating Room, Affiliated Traditional Chinese Medicine Hospital of Southwest Medical University, Luzhou, China. ${ }^{7}$ School of Chinese and Western Clinical Medicine, Southwest Medical University, Luzhou, China. ${ }^{8}$ Department of Nephropathy, Affiliated Traditional Chinese Medicine Hospital of Southwest Medical University, Luzhou, China.

Received: 23 February 2020 Accepted: 16 June 2020

Published online: 22 June 2020

\section{References}

1. Cheung KM, Karppinen J, Chan D, et al. Prevalence and pattern of lumbar magnetic resonance imaging changes in a population study of one thousand forty-three individuals. Spine. 1976;34:934-40.

2. Porto LR, Tang R, Sawka A, et al. Comparison of patient position and midline lumbar neuraxial access via statistical model registration to ultrasound. Ultrasound Med Biol. 2019;45:255-63.

3. Yan $\mathrm{S}$, Zhang $\mathrm{Y}$, Wang $\mathrm{K}$, et al. Three-dimensional morphological characteristics of lower lumbar intervertebral foramen with age. Biomed Res Int. 2018;2018:8157061.

4. Plomp KA, Dobney K, Weston DA, et al. 3D shape analyses of extant primate and fossil hominin vertebrae support the ancestral shape hypothesis for intervertebral disc herniation. BMC Evol Biol. 2019;19:226.

5. Gelalis ID, Papanastasiou El, Theodorou DJ, et al. Postoperative MRI findings 5 years after lumbar microdiscectomy. Eur J Orthop Surg Tramadol. 2019;29: $313-20$.

6. Gottlieb M, Holladay D. Ultrasound-assisted lumbar punctures: a systematic review and meta-analysis. Acad Emerg Med. 2019;26:85-96.

7. Cui X, Wang G. Radiographic anatomical relationship between spinous process and pedicle in thoracolumbar and lumbar spine. Medicine (Baltimore). 2017;96:e6732.

8. Williams $P$, Tait $G$, Wijeratne $T$. Success rate of elective lumbar puncture at a major Melbourne neurology unit. Surg Neurol Int. 2016;9:12

9. Muthusami P, Robinson AJ, Shroff MM. Ultrasound guidance for difficult lumbar puncture in children: pearls and pitfalls. Pediatr Radiol. 2017;47:82230

10. Kim HW, Ko YJ, Rhee WI, et al. Intralaminar reliability and accuracy of posterior superior iliac spine and iliac palpation for spine level estimations. J Manipulative Pbysiol Tber. 2007;30:386-9.

11. Cooperstein R. Systematic review and meta-analyses of the difference between the spinal level of the palpated and imaged iliac crests. J Can Chiropr Assoc. 2017:61:106-20.

12. Ghorbani M, Azar M, Shojaei $H$, et al. Dural arteriovenous fistulas as a relative contraindication for lumbar puncture: brief report and literature review. Br J Neurosurg. 2019;9:1-4.

13. Hosokawa $Y$, Okutomi $T$, Hyuga $S$, et al. The concordance rate of $L 3 / 4$ intervertebral lumbar level estimated by palpation and ultrasonography in Japanese parturients. J Matern Fetal Neonatal Med. 2019:1-5.

14. Harrison DE, Cailliet R, Harrison DD, et al. Reliability of centroid, Cobb, and Harrison posterior tangent methods: which to choose for analysis of thoracic kyphosis? Spine. 2001;26:227-34.

15. Yu P, Wang $Y$, Wu $X$, et al. A digital anatomic investigation of the safe triangle areas for L1-5 percutaneous minimally invasive discectomy. Surg Radiol Anat. 2020;42:103-10.

16. Cooperstein R. The reliability of palpating the posterior superior iliac spine: a systematic review. J Can Chiropr Assoc. 2016;60:36-46.
17. Konieczny MR, Reinhardt J, Prost M, et al. Signal intensity of lumbar disc herniations: correlation with age of herniation for extrusion, protrusion, and sequestration. Int J Spine Surg. 2020;14:102-7.

18. Ilyas $\mathrm{H}$, Golubovsky JL, Chen J, et al. Risk factors for 90-day reoperation and readmission after lumbar surgery for lumbar spinal stenosis. J Neurosurg Spine. 2019;31:20-6.

19. Kjaer $P$, Leboeuf-Yde C, Korsholm L, et al. Magnetic resonance imaging and low back pain in adults: a diagnostic imaging study of 40-year-old men and women. Spine. 2005:30:1173-80.

20. Fukuda K, Kawakami G. Proper use of MR imaging for evaluation of low back pain (radiologist's view). Semin Musculoskelet Radiol. 2001;5:133-6.

\section{Publisher's Note}

Springer Nature remains neutral with regard to jurisdictional claims in published maps and institutional affiliations.
Ready to submit your research? Choose BMC and benefit from:

- fast, convenient online submission

- thorough peer review by experienced researchers in your field

- rapid publication on acceptance

- support for research data, including large and complex data types

- gold Open Access which fosters wider collaboration and increased citations

- maximum visibility for your research: over $100 \mathrm{M}$ website views per year

At BMC, research is always in progress.

Learn more biomedcentral.com/submissions 